\title{
Recovery and Work Hardening Rates of an Al-10 mass \% Zn Solid Solution Alloy at Temperatures from 523 to $823 \mathrm{~K}$
}

\author{
By Takao Endo* and Norikazu Marumo**
}

\begin{abstract}
The stress and temperature dependence of steady state creep rates, recovery rates $(r=-\partial \sigma / \partial t)$ and work hardening rates $(h=\partial \sigma / \partial \varepsilon)$ were studied. The rate controlling mechanism changed from bulk to core-diffusion at about $673 \mathrm{~K}$. Application of the Yoshinaga's discriminant revealed that the effective stress level of flow stress is negligibly small.

Measurements of $r$ and $h$ were conducted by stress relaxation tests. The results were compared with a network growth theory of creep. Coincidence between the experiments and the theory was fairly good.
\end{abstract}

(Received June 15, 1981)

\section{Introduction}

The creep behaviours of solid solution alloys are classified into two classes: class I and class II. The stress exponents of class I alloys are about 3, whereas those of class II are close to 5 . The deformation mechanism of class $I$ is dragging of solute atmospheres around edge dislocations and that of class II has been believed to be the same as that of pure metals ${ }^{(1)}$.

However, recent studies on the instantaneous plastic strain with an abrupt stress change explicitly reveal that some class II alloys exhibit class I alloy behaviours ${ }^{(2)}$. It is worthwhile to note here that these exceptional class II alloys contain solute atoms with a large difference in atomic size from matrix atoms and that their effective stress levels are considerably larger than those of pure metals ${ }^{(3)-(5)}$. Since the stress exponents and steady state creep rates of $\mathrm{Al}-\mathrm{Zn}$ alloys ${ }^{(6)(7)}$ are much the same as those of pure $\mathrm{Al}$ and the size difference between $\mathrm{Al}$ and $\mathrm{Zn}$ atom is very small ${ }^{(8)}$, one naturally expects that an $\mathrm{Al}-\mathrm{Zn}$ alloy is a typical class II alloy. However, a considerable fraction of effective stress has been reported by König et $a l .{ }^{(9)}$.

* Department of Metallurgical Engineering, Faculty of Engineering, Yokohama National University, Yokohama 240, Japan.

** Graduate School, Yokohama National University, Yokohama. Present address: Isuzu Motors Ltd., Fujisawa 252, Japan.
The purpose of the present work is to elucidate the high temperature deformation mechanism of Al- $\mathrm{Zn}$ solid solution alloys.

\section{Experimental Procedures}

\section{Specimen preparation}

The purities of starting materials are 99.99 mass \% $\mathrm{Al}$ and 99.9 mass \% $\mathrm{Zn}$. For the present study, the nominal composition of $\mathrm{Al}-10$ mass $\% \mathrm{Zn}$ alloy was chosen. The cast alloy blocks were subjected to repeated cold working and anealing. The test pieces were machined into a cylindrical form $10^{-2} \mathrm{~m}$ in diameter and $2 \times 10^{-2} \mathrm{~m}$ in height. The final heat-treatments at $773 \mathrm{~K}$ for $3.6 \mathrm{ks}(1 \mathrm{~h})$ produced equiaxed grains with an average diameter of $5.3 \times 10^{-4} \mathrm{~m}$. The chemical composition of $\mathrm{Zn}$ content was 9.8 mass $\%$.

\section{Mechanical tests}

The mechanical behaviour studies were conducted by compression tests using an Instron type testing machine. A mixture of molybdenum sulfide and graphite was used as a lubricant. In a stress relaxation test, the strain increment $d \varepsilon$ is expressed by the following equation:

$$
d \varepsilon=-d \sigma / K,
$$

where $K$ is a combined Young's modulus. $K$ is given as

$$
1 / K=1 / E+(l / S)\left(1 / k_{m}\right)
$$


where $E$ is the Young's modulus of a specimen, $l$ the gage length, $S$ the cross sectional area and $k_{m}$ the spring constant of a testing machine.

$k_{m}$ was measured as a function of load by compressing the anvil without a specimen. The measured $k_{m}$ was about $30 \mathrm{MN} / \mathrm{m}$ when the applied load was greater than $2 \mathrm{kN}$. However, $k_{m}$ decreased gradually as the applied load was decreased because the testing machine did not exactly follow the Hooke's law. Consequently, $k_{m}$ values corresponding to the loads at which the stress relaxation tests were carried out were adopted in the present study. The validity for the choice of these $k_{m}$ values will be mentioned later in connection with Fig. 3.

The stress relaxation experiments were carried out by arresting the crosshead motion. The crosshead arrest characteristics were monitored using a synchroscope and a differential transformer with an accuracy of $2.5 \times$ $10^{-7} \mathrm{~m}$. No anormalies were observed in the crosshead motion.

The temperature was kept constant within $\pm 1 \mathrm{~K}$ during compression tests and within $0.25 \mathrm{~K}$ during relaxation tests.

Before the mechanical testing, each specimen was heated in the furnace for the period more than $3.6 \mathrm{ks}$ for thermal stability. There exists the strain region where plastic deformation proceeds at a constant stress over the temperature range investigated. Hereafter, we call the deformation in such a region a steady state creep.

\section{Physical Basis of Stress Relaxation Analysis}

The flow stress is generally composed of two components; effective stress $\sigma^{*}$ and internal stress $\sigma_{i}$. The effective stress is strongly dependent upon the temperature $T$ and strain rate $\dot{\varepsilon}$ while the internal stress is dependent upon the temperature only through the temperature dependence of the shear modulus.

An internal stress level is unequivocally determinable if the corresponding internal structure $\eta$ is specified. However, the effective stress is a function of $\eta$ and $\dot{\varepsilon}$ even if the testing temperature remains constant. Consequently, the flow stress is expressed as $\sigma=\sigma(\eta, \dot{\varepsilon})$.
Although $\eta$ is a path dependent quantity, a small change in $\eta$ may be expressed by eq. (3) because an infinitesimal change in $\eta$ around a certain value of $\eta$ is caused by work hardening and annealing effect.

$$
d \eta=(\partial \eta / \partial \varepsilon) d \varepsilon+(\partial \eta / \partial t) d t
$$

where $\varepsilon$ is the strain, $t$ the time and $d t=d \varepsilon / \dot{\varepsilon}$.

Abe et al..$^{(10)}$ manipulated these equations and obtained eq. (4).

$$
d \varepsilon=d \sigma /(\partial \sigma / \partial \varepsilon)_{\varepsilon}
$$

(a) In case where effective stress is appreciable, $(\partial \sigma / \partial \varepsilon)_{\varepsilon}=0$.

Eliminating $d \varepsilon$ from eqs. (1) and (4), $d \dot{\varepsilon}$ is expressed as follows, since $(\partial \sigma / \partial \varepsilon)_{\dot{\varepsilon}} \ll K$ in usual.

$$
d \dot{\varepsilon}=d \sigma /(\partial \sigma / \partial \dot{\varepsilon})_{\varepsilon}
$$

Equation (5) indicates that $d \dot{\varepsilon} \equiv\left(\dot{\varepsilon}_{r o}-\dot{\varepsilon}\right)$ approaches zero as $d \sigma$ tends to zero, where $\dot{\varepsilon}$ and $\dot{\varepsilon}_{r o}$ are the strain rates immediately before and after the stress relaxation, respectively.

(b) In case where the effective stress is negligibly small, $(\partial \sigma / \partial \dot{\varepsilon})_{\varepsilon}=0$.

In this case, the so-called Orowan's equation holds.

$$
d \sigma=h d \varepsilon-r d t
$$

where $h(\equiv \partial \sigma / \partial \varepsilon)$ and $r(=-\partial \sigma / \partial t)$ are the work hardening and recovery rates, respectively.

The steady state creep rate $\dot{\varepsilon}_{s}$ is given by eq. (7), since $d \sigma=0$ at steady states.

$$
\dot{\varepsilon}_{s}=r / h \text {. }
$$

It seems appropriate to assume that $r$ and $h$ remain unchanged just before and after the stress relaxation. Thus the strain rate immediately after the stress relaxation $\dot{\varepsilon}_{r o}$, is deduced by elimination of $d \sigma$ from eqs. (1) and (6).

$$
\dot{\varepsilon}_{r o}=r /(h+K) \text {. }
$$

Comparison between eqs. (7) and (8) leads us to conclude that $\dot{\varepsilon}_{r o}$ is always smaller than $\dot{\varepsilon}_{s}$ in case of (b) while $\dot{\varepsilon}_{r o}$ is equal to $\dot{\varepsilon}_{s}$ in case of (a).

By measuring $\dot{\varepsilon}_{r o} / \dot{\varepsilon}_{s}$, it is possible to discern whether the effective stress is appreciable or not. Hereafter, we will call $f=\dot{\varepsilon}_{\text {ro }} / \dot{\varepsilon}_{s}$ as the Yoshinaga's discriminant.

For measurements of $r$ and $h$, one obtains 
eq. (9) from eqs. (1) and (6).

$$
r=-(1+h / k) \dot{\sigma}_{r o}
$$

where $\dot{\sigma}_{r o}$ is the stress rate just after the stress relaxation. Using eqs. (6) and (9), Sakurai et $a l .^{(11)}$ obtained the following eqs. (10) and (11):

$$
\begin{aligned}
& r=-\dot{\varepsilon} \dot{\sigma}_{r o}(K+d \sigma / d \varepsilon) /\left(\dot{\varepsilon} K+\dot{\sigma}_{r o}\right) \\
& h=K\left(\dot{\varepsilon} d \sigma / d \varepsilon-\dot{\sigma}_{r o}\right) /\left(\dot{\varepsilon} K+\dot{\sigma}_{r o}\right)
\end{aligned}
$$

\section{Experimental Results}

\section{Temperature and stress dependences of steady state creep rates}

The temperature and stress dependences of steady state creep rates are shown in Figs. 1 and 2 , respectively, where $E$ is the Young's modulus of $\mathrm{Al}$ at testing temperatures ${ }^{(12)}$. Figures 1 and 2 indicate that the steady states are expressed in the following form:

$$
\dot{\varepsilon}_{s} \propto(\sigma / E)^{n_{s}} \exp \left(-Q_{c} / R T\right),
$$

where $\sigma$ is the stress, $n_{s}$ the stress exponent, $Q_{c}$ the activation energy for creep and $R T$ has usual meanings.

Figure 2 shows that the activation energies for creep above and below $673 \mathrm{~K}$ are $136 \mathrm{~kJ} / \mathrm{mol}$ and $106 \mathrm{~kJ} / \mathrm{mol}$, respectively. These activation energies coincide with those for creep of $\mathrm{Al}^{(1)}$ in bulk and core-diffusion controlled ranges, respectively.

In accordance with the change in the deformation mechanism, the stress exponents

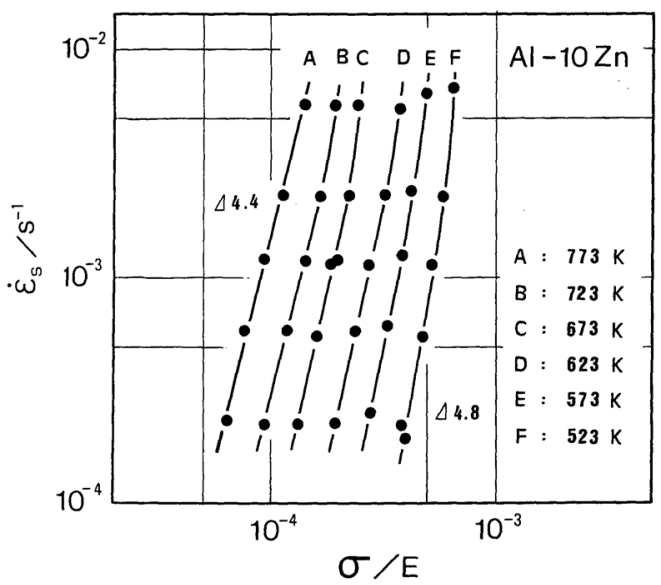

Fig. 1 Stress dependence of steady state creep rates.

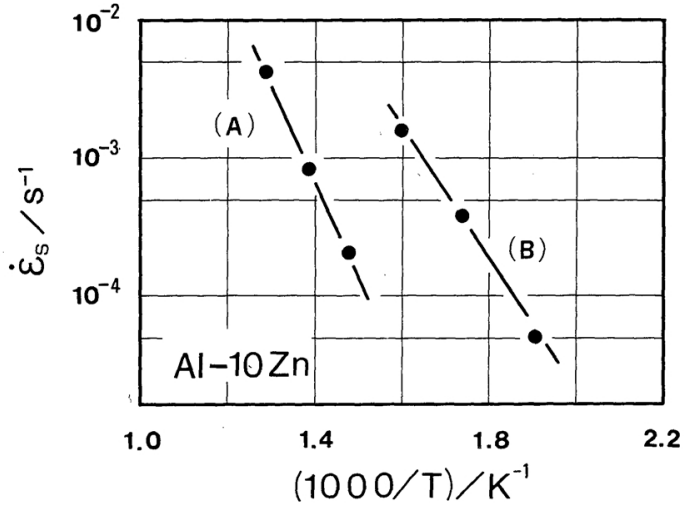

Fig. 2 Temperature dependence of steady state creep rates. (A) and (B) are under $\sigma / E=3.0 \times 10^{-4}$ and $\sigma / E=1.3 \times 10^{-4}$, respectively.

change from 4.4 at higher temperatures to 4.8 at lower temperatures. The transition temperature $T_{c}$ observed in the present study seems rather higher. This is presumably because the strain rates used in this study are somewhat higher than those usually reported ${ }^{(13)}$. The stress exponents determined in the present study agree well with those reported by other investigators for various $\mathrm{Al}-\mathrm{Zn}$ alloys with different zinc contents ${ }^{(6)(7)}$.

\section{Ratio of internal stress to total flow stress}

Various techniques such as stress $\operatorname{dip}^{(14)}$ or strain $\operatorname{dip}^{(15)}$ have been proposed for determination of internal stress levels. It should be, however, noticed that the direct application of these techniques leads to an underestimation of internal stress levels ${ }^{(16)}$ because the rate of decrease in dislocation density just after stress drop is unexpectedly large $e^{(17)}$ and the time required for the measurement of an internal stress level (usually 20 to 30 seconds) is too long to neglect the decrease in internal stress during the measurements.

An improvement for the conventional stress dip techniques has been proposed ${ }^{(16)}$. The new technique is sometimes called as a back extrapolation method in which the decrease in internal stress during testing is attempted to be eliminated.

Using a similar type of the technique, the internal stress levels of Al-10 mass $\% \mathrm{Zn}$ alloy were turned out to be almost the same as the flow stress. The results will be written else- 
where $^{(18)}$. In the present study, the Yoshinaga's discriminant will be applied in order to envisage whether or not the effective stress level of the Al-Zn alloy is appreciable.

Figure 3 is the relation between $\dot{\varepsilon}_{s}$ and $\dot{\varepsilon}_{r o}$. A prominent feature of Fig. 3 is that $\dot{\varepsilon}_{r o}<\dot{\varepsilon}_{s}$ in $\mathrm{Al}$ and the $\mathrm{Al}-\mathrm{Zn}$ alloy whereas $\dot{\varepsilon}_{r o}=\dot{\varepsilon}_{\mathrm{s}}$ in the Al$\mathrm{Mg}$ alloy.

Detail studies on high concentration $\mathrm{Al}-\mathrm{Mg}$ alloys revealed that the rate controlling mechanism is the dragging motion of solute atmospheres around edge dislocation ${ }^{(19)(20)}$ and the fraction of effective stress is appreciable ${ }^{(16)}$. The coincidence of $\dot{\varepsilon}_{s}$ with $\dot{\varepsilon}_{r o}$ in the Al-Mg alloy explicitly indicates that the Yoshinaga's discriminant is valid and that the $K$ or $k_{m}$ value adopted is passable for the present study.

On the other hand, $\dot{\varepsilon}_{r o} / \dot{\varepsilon}_{s}$ for Al or the Al-Zn alloy is smaller than unity. Toma et al. ${ }^{(16)}$ clearly demonstrated that the effective stress level of $\mathrm{Al}$ is negligibly small using the back extrapolation technique. This is a further support for the validity of the Yoshinaga's discriminant. Based on these facts it may be safely concluded that the effective stress of the $\mathrm{Al}-\mathrm{Zn}$ alloy is negligibly small.

The present conclusion is inconflict with that obtained by König et al. ${ }^{(9)}$. They reduced the time interval necessary for determining the sign of creep rate after the stress drop up to $2 \mathrm{~s}$. This time interval is about one-tenth of that needed by Alhquist et al. in the course of studies for Al. Notwithstanding the large difference in the time interval, the internal stress levels determined by König et al. are about $50 \%$, and they are much the same as those determined by Alhquist et al.

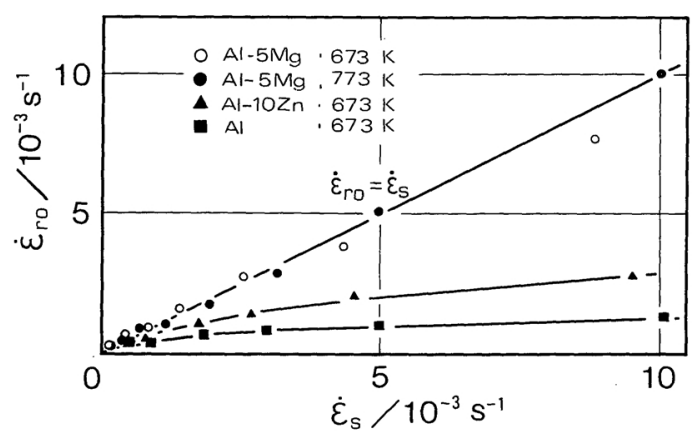

Fig. 3 Relation between $\dot{\varepsilon}_{r o}$ and $\dot{\varepsilon}_{s}$.
It is difficult to make clear the reasons for the disparity in question. However, the disparity suggests that the change in dislocation density just after the stress reduction is unexpectedly large ${ }^{(17)}$ and not only the dislocation density but also the dislocation configurations, which are more changeable than the former, are important for internal stress levels.

\section{Measurements of $\boldsymbol{r}$ and $\boldsymbol{h}$}

The high temperature deformation mechanism of the $\mathrm{Al}-10$ mass $\% \mathrm{Zn}$ alloy is recovery controlled since its effective stress is negligible. Therefore, eqs. (10) and (11) are applicable for the measurements of $r$ and $h$ during steady states.

Figures 4 and 5 are respectively the stress and temperature dependences of recovery rates, in which $E$ is the Young's modulus of $\mathrm{Al}$ at testing temperatures. From Figs. 4 and 5, the

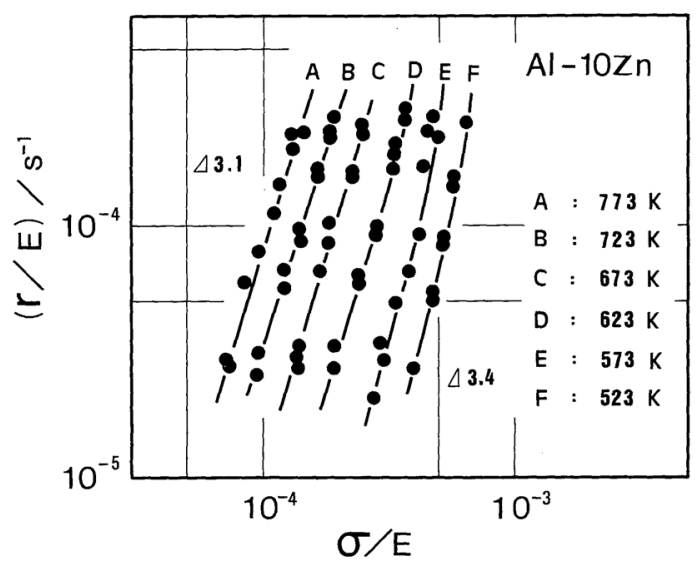

Fig. 4. Stress dependence of recovery rates.

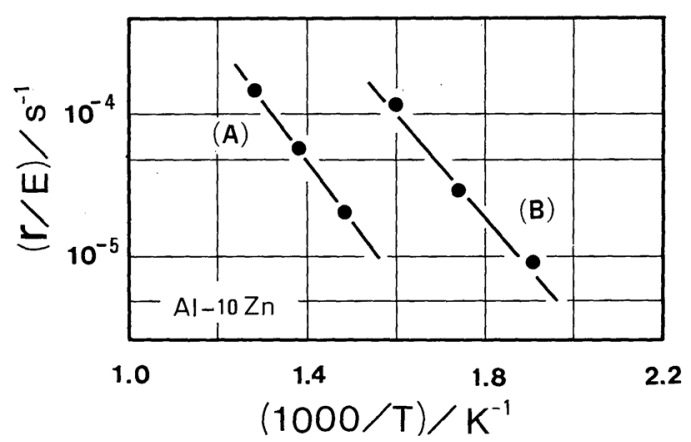

Fig. 5 Temperature dependence of recovery rates. (A) and (B) are under $\sigma / E=1.5 \times 10^{-4}$ and $\sigma / E=$ $3.6 \times 10^{-4}$, respectively. 
recovery rates of structures are empirically expressed in the following form:

$$
r / E \propto(\sigma / E)^{n_{r}} \exp \left(-Q_{r} / R T\right),
$$

where $n_{r}$ is the stress exponent and $Q_{r}$ the apparent activation energy for the recovery process.

Since the dislocation density or the subgrain size has been reported to be independent of test temperatures ${ }^{(1)(22)(23)}, Q_{r}$ can be determined assuming that the internal structures are identical when compared at the same $\sigma / E$ values. $Q_{r}$ values obtained from Fig. 5 are 63.5 and $87.8 \mathrm{~kJ} / \mathrm{mol}$ above and below $T_{c}$, respectively.

Although one expects that $Q_{r}$ coincides with $Q_{c}, Q_{r}$ values are smaller than $Q_{c}$ in both regions. The reason for the discrepancy between them will be discussed later.

Figures 6 and 7 are the stress and temperature dependences of work hardening rates, respectively. As in the case of $r, h$ is expressed as

$$
h / E \propto(\sigma / E)^{n_{h}} \exp \left(-Q_{n} / R T\right),
$$

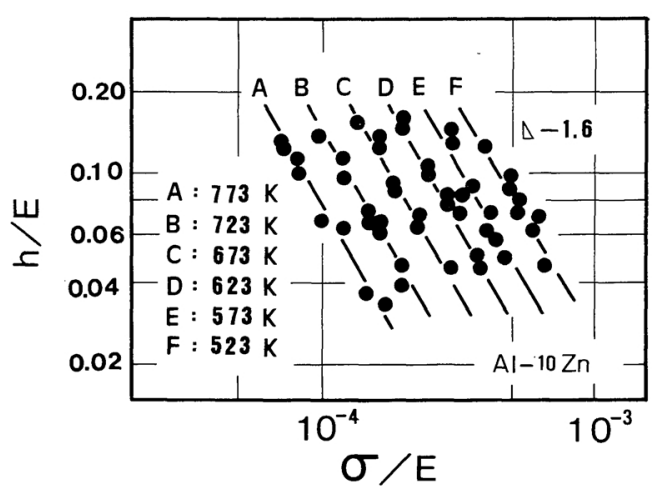

Fig. 6 Stress dependence of work hardening rates.

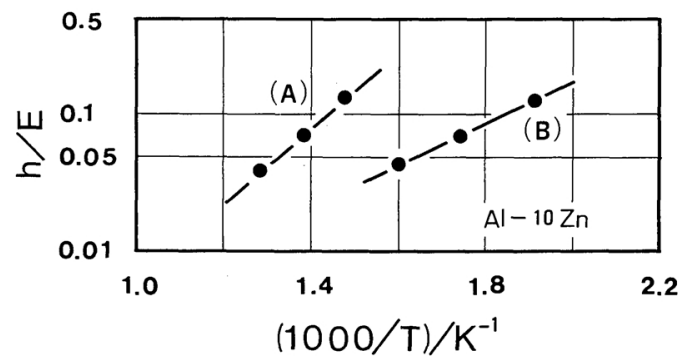

Fig. 7 Temperature dependence of work hardening rates. (A) and (B) are under $\sigma / E=2.0 \times 10^{-4}$ and $\sigma / E=3.5 \times 10^{-4}$, respectively. where $n_{h}$ is the stress exponent for work hardening rates and $Q_{h}$ the parameter describing the temperature dependence. The various parameters determined in the present study are listed in Table 1.

Although $Q_{h}$ values above and below $T_{c}$ differ from each other, $n_{h}$ is a constant over a wide range of temperature. This is in contrast to that of $n_{r}$. Comparison among $n_{s}, n_{r}$ and $n_{h}$ reveals that the change in $n_{s}$ above and below $T_{c}$ reflects the change in the stress exponents for recovery.

\section{Discussion}

\section{Temperature dependence of recovery rates}

Mitra et al. ${ }^{(24)}$ measured the recovery rates by means of a zero creep rate method. They obtained the activation energy for recovery smaller than that for creep although they did not mention the reason for this at all. Another example was given by Sakurai et al. ${ }^{(11)}$. They measured the recovery rates for $\mathrm{Al}$ from the stress relaxation analysis and showed that $Q_{r}$ was smaller than $Q_{c}$. Goto et al. ${ }^{(25)}$ studied the recovery rates in somewhat different manner and also found the same trend. The test temperatures of some earlier works may override the transition temperature from core to bulkdiffusion controlled range. In contrast to earlier works, $Q_{r}$ values in the present study were determined separately in both regions. It was confirmed that $Q_{r}$ is smaller than $Q_{c}$ in both regions. Based on these experimental facts, it follows that the apparent activation energy for recovery measured in this manner is smaller than that for creep.

Above $T_{c}$, the rate controlling mechanism for class II alloys is that involving the bulkdiffusion, because $Q_{c}$ is almost equal to the activation energy for self-diffusion ${ }^{(1)}$ and the activation volumes for creep coincide well with

Table 1 Parameters describing steady states.

\begin{tabular}{ccccccc}
\hline $\begin{array}{c}\text { Temp. } \\
(\mathrm{K})\end{array}$ & $n_{s}$ & $n_{r}$ & $n_{h}$ & $Q_{c}$ & $\begin{array}{c}Q_{r} \\
(\mathrm{~kJ} / \mathrm{mol})\end{array}$ & $Q_{h}$ \\
\hline $523-623$ & 4.8 & 3.4 & -1.6 & 106 & 63.5 & -27.2 \\
$673-773$ & 4.4 & 3.1 & -1.6 & 136 & 87.8 & -50.2 \\
\hline
\end{tabular}


those for self-diffusion ${ }^{(26)}$. Therefore, we must seek out the reasons why $Q_{r}$ is smaller than $Q_{c}$.

So far, the internal structures have been assumed to be independent of the temperature when compared at the same $\sigma / E$ since the dislocation density or subgrain size is strongly dependent on stress but independent of temperatures $^{(22)(23)}$. However, some recent studies suggest that the internal structures are dependent upon the temperature ${ }^{(21)}$.

Figure 8 is a schematic illustration of the annealing effect at a test temperature upon recovery rates ${ }^{(21)} \cdot r_{0}$ and $r_{t}$ in the figure are recovery rates before and after the annealing, respectively and they were measured at the same temperature and stress. It can be seen that the ratio of recovery rates, $r_{t} / r_{0}$ decreases at first and then increases gradually up to unity.

The decrease in rates of recovery by the annealing appears to indicate that the deformed structures change into more stable configurations by the annealing. Such configurations inevitably diminish the recovery rates. This trend must be enhanced as the annealing temperatures are raised. Although the argument developed here is not quantitative, however, the reason why $Q_{r}$ is smaller than $Q_{c}$ is easily understood.

\section{Rate of recovery}

The rate of recovery, $r(=-\partial \sigma / \partial t)$, is expressed as

$$
r=-(\partial \rho / \partial t)(\partial \sigma / \partial \rho)
$$

where $\rho$ is the total dislocation density. The loss of total dislocation density is generally given by the following equation ${ }^{(27)}$ :

$$
-(\partial \rho / \partial t)=\rho_{c}\left(v_{c} / d\right)
$$

where $\rho_{c}$ is the density of climbing dislocations,

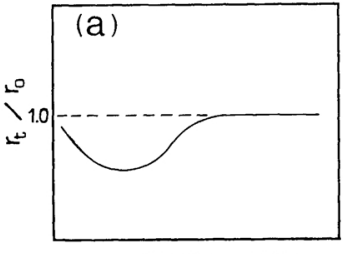

ANNEALING TIME

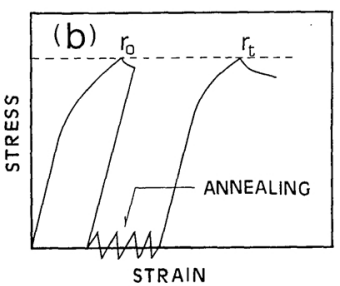

Fig. 8 Schematic illustration of annealing effect upon $r_{t} / r_{\mathrm{o}}$ (a) and procedures for determination of $r_{\mathrm{o}}$ and $r_{t}(\mathrm{~b})$. $v_{c}$ the rate of climb and $d$ the climb distance for the loss of dislocations.

Various types of recovery models ${ }^{(24)(28)-(30)}$ have been proposed. Among these the three dimensional network growth model is most compatible with the direct observation of crept specimens $^{(31)}$. Therefore, comparison of experiments with a network theory will be pushed forward.

A network growth theory is based on the view that dislocations within subgrains exist as a three dimensional network. Deformation is considered to cause refinement of the network. Mitra et al. ${ }^{(24)}$ applied the Friedel's network growth theory ${ }^{(32)}$ to explain the stress dependence of recovery rates. There exist some criticisms ${ }^{(11)(17)(31)}$ against their treatments, because the Friedel's theory deals with the network growth in a static process while the network growth during creep is in a dynamic nature.

Evans et al. ${ }^{(33)}$ have recently studied the growth of dislocation network in which they have taken into account three components for the climbing force; (i) the contribution of applied stress (ii) the elastic interaction between links and (iii) the changes in line energy. Based on their analysis, the climb process will be assumed to be added by a simple sum of these components. Then overall climb force per unit length $F$ is given as

$$
F=\left[\sigma b / 2+G b^{2} / 2 \pi \bar{x}(1-v)+G b^{2} / \bar{x}\right],
$$

where $\bar{x}$ is an average mesh size of the network. The first term in the brackets is due to the applied stress, the second term is due to the elastic interaction force and the last term is due to the dislocation line energy. Taking $v$ as 0.3 , $F$ is of the order of $1.74 \sigma b$.

The climbing rate of link dislocation, $v_{c}$, is given by the following equation ${ }^{(33)}$ :

$$
v_{c}=2 \pi D_{L} F b / k T \ln (G / 2 \sigma),
$$

where $D_{L}$ is the lattice self-diffusion coefficient. Assuming that $\bar{x}=1 / \sqrt{\rho}, \rho_{c}=\rho$ and $d=\bar{x}$, one obtains $r$ using eqs. (15), (16), (17) and (18) as

$$
r=3.5 \pi D_{L} b \sigma^{3} / G k T \ln (G / 2 \sigma) .
$$

Substituting $b, G^{(34)}$ and $D_{L}^{(35)}$ into eq. (19), $r$ values can be calculated. 


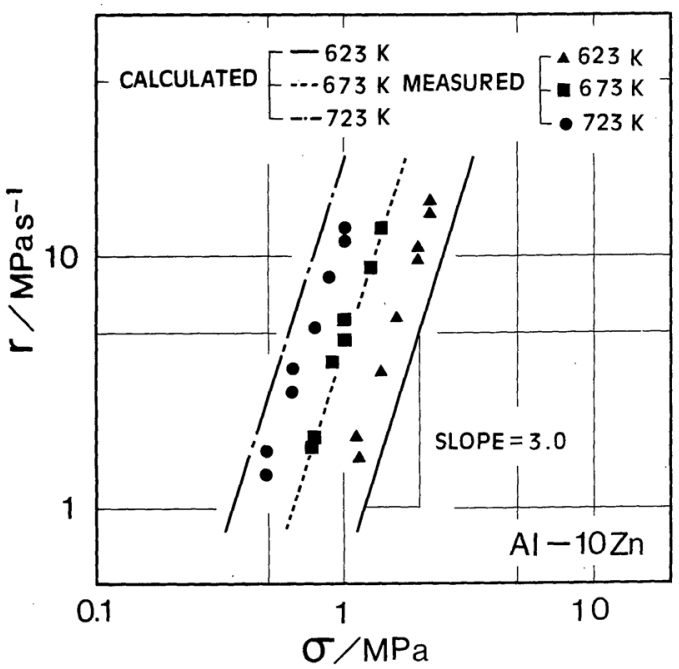

Fig. 9 Comparison of recovery rates with eq. (19).

Figure 9 is comparison of the experiments with the results of calculation. In deducing eq. (19), the density of thermal jogs is omitted although Gittus ${ }^{(36)}$ takes into account. This is based on the experimental facts that the activation energy for creep is much the same as that for self-diffusion. This implies that the thermal jogs are not important during creep.

It can be seen from Fig. 9 that the network growth theory for creep gives the reasonable recovery rates during steady state creep deformation.

\section{Rate of work hardening}

The strain rate is given by eq. (20) when dislocations travel in a free flight manner.

$$
\dot{\varepsilon}=\phi \dot{N} b \bar{A},
$$

where $\phi$ is a conversion factor from shear to tensile strain $(\phi \simeq 1 / 2), \dot{N}$ the generation rate of dislocation loops per unit volume and $\bar{A}$ is an average area swept out by the dislocation loops.

Since the structures remain constant during steady states, the strain increment is expressed as

$$
\delta \varepsilon=\phi b \bar{A} \delta N,
$$

where $N$ is the number of dislocation loops per unit volume. Thus,

$$
h=1 /[\phi b \bar{A}(\partial N / \partial \sigma)] .
$$

Assuming that $\bar{A}$ is a circle with radius $R$
$\left(\bar{A}=\pi R^{2}\right)$, the increment in dislocation density is given as

$$
\delta \rho=2 \pi R \delta N
$$

Assuming again $\sigma \cong G b \sqrt{\rho}$, eq. (24) results from eqs. (22) and (23).

$$
h=G^{2} b / \phi R \sigma .
$$

If $R$ is of the order of mesh size $(\bar{x})$, eq. (25) results, while if $R$ is of the order of subgrain size $(D)$, eq. (26) results.

$$
\begin{aligned}
& h \cong 2 G . \\
& h \cong 2 G^{2} b / D \sigma .
\end{aligned}
$$

Equation (25) was deduced by Alden ${ }^{(27)}$ and Evans $^{(37)}$ in a similar manner and eq. (26) is analogous to the equation obtained by Evans ${ }^{(38)}$ using the mesh length theory ${ }^{(39)}$.

The stress dependence of subgrain size is expressed in the form:

$$
D=C G b / \sigma
$$

where $C$ is a constant. The experimentally determined $C$ values $^{(40)-(43)}$ are between 19.4 and 78 . The stress dependence reported by Orlova et al. ${ }^{(44)}$ is somewhat different from eq. (27). Their results are approximately expressed as

$$
D=\left(C^{\prime} G b / \sigma\right)^{1 / 2},
$$

where $C^{\prime}$ is $98 \mu \mathrm{m}$.

Ferreira et al. $^{(42)}$ mentioned that eq. (28) seems dubious although they obtained a similar relation at low stresses. The reason why they felt eq. (28) dubious is based on their experimental fact that the fine substructures were localized in certain regions of the foils. Such a morphology is not typical when foils are carefully prepared from crept specimens. The work hardening rates were calculated using various $D$ vs $\sigma$ relations. The results are shown in Fig. 10.

Lagneborg $^{(45)}$ deduced the work hardening rates using a $\rho$ vs $\sigma$ relation obtained empirically at room temperature where the recovery process is not so important. It has often been found that the dislocation density is expressed in the following form ${ }^{(46)}$ :

$$
\rho=M \varepsilon,
$$




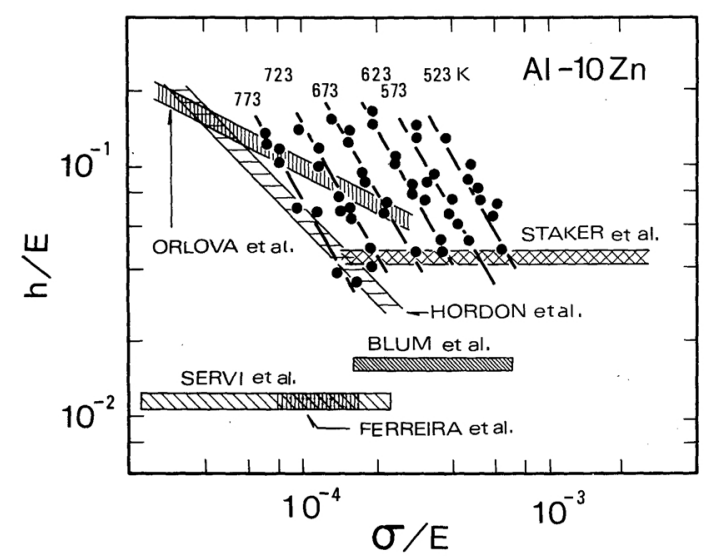

Fig. 10 Comparison of work hardening rates with eqs. (26) and (30). The names described in the figure indicate the investigators who determined the experimental constants appearing in eq. (27), (28) or (29).

where $M$ is a constant. It is interesting to note that $M$ values are about the same for different materials $^{(46)}$.

With eq. (29), the work hardening rate is expressed as

$$
h=M G^{2} b^{2} / 2 \sigma .
$$

Using $M$ for $\mathrm{Al}^{(47)}$, the work hardening rates can be calculated. The results are also shown in Fig. 10. The results from eq. (25) are not shown since they are considerably greater than the experimental data.

The work hardening models here discussed are simple. However, they give the correct order of magnitude for $h$ values. As for the stress dependence, eqs. (25) and (26) can not explain the experiments although they are tangible in relation to the network growth theory. On the other hand, the stress dependence of eq. (30) is in accordance with the experiments though eq. (29) is not physically related to the network growth theory. Further studies are desirable.

As already mentioned, the experimental data show that the work hardening rates at a given stress are temperature dependent although any work hardening models do not involve temperature dependent terms. This is probably because we postulated that the internal structures are independent of testing temperatures and that the deformed substructures are identical under the same stress levels. Although the direct observations so far reported fail to reveal the temperature dependence of dislocation structures, the present and also some recent studies clearly indicate that fine structures such as dislocation arrangements and the nature of sub-boundaries are dependent on testing temperatures.

\section{Conclusions}

The compression tests and stress relaxation analysis at temperatures from 523 to $823 \mathrm{~K}$ lead to the following conclusions:

(i) The effective stress of the Al-10 mass $\% \mathrm{Zn}$ alloy is negligible;

(ii) The stress and temperature dependence of steady state creep rates $\left(\dot{\varepsilon}_{s}\right)$, recovery rates $(r)$ and work hardening rates $(h)$ are expressed by eqs. (12), (13) and (14) in the order of its appearance. The parameters describing $\dot{\varepsilon}_{s}, r$ and $h$ are listed in the Table 1;

(iii) The coincidence of $r$ or $h$ with a network growth theory of creep is fairly good.

\section{REFERENCES}

(1) O. D. Sherby and P. M. Burke: Prog. Mater. Sci., Ed. B. Chalmers and W. Hume-Rothery, 13 (1966), 325.

(2) H. Oikawa: Phil. Mag., 37 (1978), 707.

(3) H. Oikawa, J. Kariya and S. Karashima: Met. Trans., 9A (1978), 1281.

(4) S. Kikuchi, J. Shimizu and M. Adachi: J. Japan Inst. Metals, 41 (1977), 182 (in Japanese).

(5) H. Oikawa, K. Sugawara and S. Karashima: Trans. JIM, 19 (1978), 611.

(6) T. G. Langdon, R. B. Vastava and P. Yavari: ICSMA 5, 1 (1979), 271.

(7) T. Endo, T. Nomura, T. Enjyo and M. Adachi: J. Japan Inst. Metals, 35 (1971), 427 (in Japanese).

(8) J. E. Dorn, D. Pietrokowsky and T. E. Tietz: Trans. AIME, 188 (1950), 933.

(9) G. König and W. Blum: Acta Met., 25 (1977), 1531.

(10) M. Abe, H. Yoshinaga and S. Morozumi: J. Japan Inst. Metals, 40 (1976), 393 (in Japanese).

(11) S. Sakurai, M. Abe, H. Yoshinaga and S. Morozumi: J. Japan Inst. Metals, 42 (1978), 432 (in Japanese).

(12) W. Köster: Z. Metallk., 39 (1948), 145.

(13) H. Luthy, A. K. Miller and O. D. Sherby: Acta Met., 28 (1980), 169.

(14) A. A. Solomon: Rev. Sci. Instrum., 40 (1969), 1025.

(15) C. N. Alhquist and W. D. Nix: Scripta Met., 3 (1969), 679. 
(16) K. Toma, H. Yoshinaga and S. Morozumi: J. Japan Inst. Metals, 38 (1974), 170 (in Japanese).

(17) J. Hausselt and W. Blum: Acta Met., 24 (1976), 1027.

(18) T. Endo and N. Marumo: unpublished.

(19) R. Horiuchi and H. Yoshinaga: Trans. JIM, 6 (1965), 131.

(20) R. Horiuchi and M. Otsuka: Trans. JIM, 13 (1972), 284.

(21) T. Endo: Kouon henkei to hakai, Iron and Steel Inst. Japan, (1981), 20 (in Japanese).

(22) A. K. Mukherjee: Trieties on Materials Sci. and Tech., Ed. by R. J. Arsenault, Academic Press, 6 (1975), p. 161.

(23) H. Oikawa and A. Yasuda: Metal Sci., 19 (1979), 551.

(24) S. K. Mitra and D. McLean: Proc. Roy. Soc. A295 (1966), 288.

(25) A. Goto, H. Oikawa and S. Karashima: Trans. JIM, 21 (1980), 15.

(26) O. D. Sherby and J. Weertman: Acta Met., 27 (1979), 387.

(27) T. H. Alden: Acta Met., 17 (1969), 1435.

(28) J. Weertman: J. Appl. Phys., 28 (1957), 362.

(29) F. R. N. Nabarro: Phil. Mag., 16 (1967), 237.

(30) W. Blum: Phys. Status Solidi (b) 45 (1971), 561.

(31) S. Takeuchi and A. S. Argon: J. Mater. Sci., 11 (1976), 1542.

(32) J. Friedel: Dislocations, Pergamon Press, (1964), p. 239.

(33) H. E. Evans and G. Knowles: Acta Met., 25 (1977), 963.

(34) H. B. Huntington: Solid St. Phys., 7 (1958), 213.

(35) T. S. Lundy and J. F. Murdock: J. Appl. Phys., 38 (1962), 1671.

(36) J. H. Gittus: Acta Met., 22 (1974), 789.

(37) H. E. Evans: Phil. Mag., 28 (1973), 227.

(38) W. J. Evans: Metal Sci., 10 (1976), 170.

(39) D. Kuhlman-Wilsdorf: Met. Soc. Conf. Work Hardening, Ed. by J. P. Hirth and J. Weertman, 46 (1966), 97.

(40) M. R. Staker and D. L. Holt: Acta Met., 20 (1972), 569.

(41) I. S. Servi and N. J. Grant: Trans. AIME, 191 (1951), 917.

(42) I. Ferreira and R. G. Stang: Mater. Sci. Eng., 38 (1979), 169.

(43) W. Blum, A. Absenger and R. Feilhuer: ICSMA 5, 1 (1978), 265.

(44) A. Orlovă, Z. Tobolovǎ and J. Čadek: Phil. Mag., 26 (1972), 1263.

(45) R. Lagneborg: Mater. Sci. J., 3 (1969), 161.

(46) A. S. Keh and S. Weisman: Electron Microscopy and Strength of Crystals, Ed. by G. Thomas and J. Washburn, (1963), p. 231.

(47) M. J. Hordon and B. L. Averback: Acta Met., 9 (1961), 247. 